\title{
Re: A Randomized Clinical Trial Comparing Nitrofurazone-coated and Uncoated Urinary Catheters in Kidney Transplant Recipients: Results from a Pilot Study
}

\author{
Menezes FG1, Corrêa L2, Medina-Pestana J03, Aguiar WF4 \\ 1 Israelita Albert Einstein Hospital, Clinic of Infection Control and Prevention, São Paulo, Brazil \\ 2Universidade Federal de São Paulo Faculty of Medicine, Department of Infectious Diseases, São Paulo, Brazil \\ 3 Universidade Federal de São Paulo, Hospital do Rim, Nephrology Division, São Paulo, Brazil \\ 4Universidade Federal de São Paulo, Hospital do Rim, Department of Urology, São Paulo, Brazil
}

Transpl Infect Dis 2019;21:e13031. doi: 10.1111/tid.13031.

\section{EDITORIAL COMMENT}

Urinary tract infections (UTIs) are the most common bacterial infections observed after kidney transplantation. Urinary catheters impregnated with antimicrobials, can inhibit or delay pathogen colonization and may contribute to infection prevention. However most studies comparing catheters impregnated with antimicrobials exclude immunosuppressed patients, such as kidney transplant recipients. In this pilot, single-centre, open-label, randomised controlled trial authors have tested whether Nitrofurazone-coated silicone urinary catheters reduced asymptomatic bacteriuria and UTIs in adult living donor kidney transplant recipients. Two hundred fourteen living donor kidney transplant recipients were randomised in a 1:1 ratio with a computer-generated system to a Nitrofurazone-coated silicone urinary catheter and non-impregnated silicone urinary catheter of whom 176 completed the study. There were no differences between groups in the reduction of asymptomatic bacteriuria and UTIs. Patients reported higher levels of discomfort and side effects with the use of Nitrofurazone-coated catheter ( $46.6 \%$ vs $38.9 \%, p=0.007$ ). The results of this study do not suggest the use of Nitrofurazone-coated silicone urinary catheters in this population.

Yarkın Kamil YAKUPOGLU, MD

๑Copyright 2019 by the Association of Urological Surgery / Journal of Urological Surgery published by Galenos Publishing House. 